\title{
Mapping Galactic spiral arm structure: the IPHAS survey and Virtual Observatory access
}

\author{
Nicholas A. Walton ${ }^{1}$, Janet E. Drew ${ }^{2}$, Eduardo A. Gonzalez-Solares ${ }^{1}$, \\ Robert Greimel $^{3}$, Ella C. Hopewell ${ }^{2}$ and Mike J. Irwin ${ }^{1}$ \\ ${ }^{1}$ Institute of Astronomy, University of Cambridge, Madingley Road, Cambridge CB3 0HA, UK \\ email: naw,eglez@ast.cam.ac.uk \\ ${ }^{2}$ Astrophysics Group, Imperial College London, Prince Consort Road, London SW7 2BZ, UK \\ email: j.drew,e.hopewell@imperial.ac.uk \\ ${ }^{3}$ Isaac Newton Group of Telescopes,
}

Apartado de corras 321, E-38700 Santa Cruz de La Palma, Tenerife, Spain

email: greimel@ing.iac.es

There is now considerable interest in how stellar streams in the Milky Way can be used to probe how the earlier merger history of our galaxy, which in turn can be related to hierarchical models of galaxy evolution.

One example stream is the Sagittarius stream, caused by the disruption of the Sagittarius Dwarf galaxy. Related to this is the ability to accurately map the major spiral arms in our galaxy. There is much uncertainty, with Russeil (2003) providing a recent spiral arm model. Early type emission line stars are an easily identified class of stars which can be (and have been) utilised effectively to map spiral arm structure.

We report on the use of IPHAS (<http://www.iphas.org >) (the INT/WFC Photometric $\mathrm{H} \alpha$ Survey of the Northern Galactic Plane (Drew et al. 2005) carried out in the $\mathrm{H} \alpha, r^{\prime}$ and $i^{\prime}$ bands to a depth of $\sim 20 \mathrm{mag}$ in $r^{\prime}$ ) as a rich source of these emission line stars. This paper includes a pilot mapping of Galactic spiral arm structure at $l \simeq 250^{\circ}$, based on the older Schmidt $\mathrm{H} \alpha$ survey where the candidate tracer stars have been selected via their colours - and then confirmed via multi-object spectroscopy. Analysis of these data underline how the increased numbers of fainter tracer stars available from IPHAS, have the potential to pin down spiral arm structure in the outer Galaxy.

We describe how the AstroGrid Virtual Observatory system has been utilised to provide access to the IPHAS data products (see <http://www. astrogrid.org $>$ ). This is supporting the analysis of the large pipeline processed data products, being analysed by members of the IPHAS consortium. We note both the deployment of Virtual Observatory components in making the data available through the VO, but also how the AstroGrid system is being used by the astronomer, for instance in the use of workflows to automate certain routine analysis operations, such as generating lists of candidate emission line stars, based on database queries of the IPHAS catalogue data.

\section{References}

Drew, J. E., Greimel, R., Irwin, M. J., et al. 2005, MNRAS, 362, 753

Russeil, D. 2003, A\&EA, 397, 133 